\title{
Low muscle mass impairs fat utilization during light-intensity exercise in Chinese men
}

\author{
Lei Chen ${ }^{1 \#}$, Tingting Tang ${ }^{2 \#}$, Juan Liu ${ }^{1}$, Jing $\mathrm{Yu}^{1}$, Wenjuan $\mathrm{Di}^{1}$, Yusheng $\mathrm{Li}^{3}$, Shan $\mathrm{Lv}^{1}$ \\ ${ }^{1}$ Department of Geratology, the First Hospital Affiliated to Nanjing Medical University, Nanjing, China; ${ }^{2}$ Department of Geratology, the First \\ People's Hospital of Yancheng, Yancheng, China; ${ }^{3}$ Deparment of Orthopedics, Xiangya Hospital, Central South University, Changsha, China \\ Contributions: (I) Conception and design: L Chen; (II) Administrative support: J Yu; (III) Provision of study materials or patients: None; (IV) \\ Collection and assembly of data: T Tang; (V) Data analysis and interpretation: S Lv, Y Li; (VI) Manuscript writing: All authors; (VII) Final approval \\ of manuscript: All authors. \\ "These authors contributed equally to this work. \\ Correspondence to: Dr. Yusheng Li. Department of Orthopedics, Xiangya Hospital, Central South University, Changsha, China. \\ Email: liyusheng@csu.edu.cn; Dr. Shan Lv. Department of Geriatric Endocrinology, The First Affiliated Hospital of Nanjing Medical University, \\ 300 Guangzhou Road, Nanjing, China. Email: lvshan3366@126.com.
}

\begin{abstract}
Background: Age-related loss of the muscle mass is linked to obesity and diabetes because the muscle is the major site for fat oxidation which is influenced by exercise. We aimed to explore the association between fat oxidation rates (FORs) and exercise intensity during age-related muscle loss.

Methods: A total of 224 healthy Chinese men aged 23-92 years were recruited. Fat and lean tissue masses were measured by DXA. The subjects were also tested using graded incremental exercise with an increased intensity of $10 \mathrm{~W}$ until maximal fat oxidation rate $\left(\mathrm{Fat}_{\max }\right)$ was achieved. Real-time contribution (\%) of fat to total energy expenditure were determined using indirect calorimetry.

Results: We found that appendicular skeletal muscle mass/weight (ASM/Wt) decreased during ageing and positively correlated with FORs during low-intensity exercise. In multiple linear regression models, FORs was positively related with ASM/Wt $(\beta=0.446, \mathrm{P}=0.0091)$ but negatively associated with exercise intensity ( $\beta=-0.573, \mathrm{P}<0.0001$ ), whereas fat oxidation rate did not show any association with age. Moreover, there was a significantly negative correlation between ASM/Wt and $\mathrm{Fat}_{\max }$.

Conclusions: Our findings highlight the importance of muscle mass in fat utilization during low-intensity exercise. A higher exercise intensity indicated by $\mathrm{Fat}_{\max }$ is recommended for improving fat oxidation in Chinese men with decreased muscle mass.
\end{abstract}

Keywords: Muscle mass; exercise intensity; fat utilization; ageing

Submitted Mar 11, 2020. Accepted for publication Jun 10, 2020.

doi: 10.21037/apm-20-611

View this article at: http://dx.doi.org/10.21037/apm-20-611

\section{Introduction}

Skeletal muscle comprises about $40 \%$ of total body mass in a healthy-weight individual (1). Ageing is associated with body composition changes, with increased visceral fat and reduced muscle mass (2). Sarcopenia is defined as the loss of muscle mass and strength due to the ageing, and is associated with insulin resistance and metabolic syndrome. The changes in skeletal muscle are especially important because muscle is the major site for substrate utilization (3). Previous studies have indicated that skeletal muscle and the heart consume around $30 \%$ of resting energy consumption and almost $100 \%$ of increased energy during exercise (4). Moreover, carbohydrate $(\mathrm{CHO})$ and fat provide the vast majority of fuel required by skeletal muscle (5).

The ageing muscle is characterized by fat infiltration (6). Thus, the accumulation of lipids within the muscles is an important factor leading to insulin resistance (7). 
A research on obesity men showed that the exercise eliciting the maximal fat oxidation but not high-intensity interval training reduced insulin resistance significantly (8). In addition, previous studies have reported that fat oxidation rates (FORs) which predominantly contribute to energy expenditure is influenced by several factors, such as muscle mass, activity state, exercise intensity, exercise mode and nutrition $(9,10)$.

Exercise has been explored as one of the most important factor influencing fat utilization. In general, the fractional contribution of $\mathrm{CHO}$ to the fuel supply is small at low intensity and rises with the exercise intensity increase (11), whereas FORs reaches its peak at moderate exercise intensities normally ranging between $55 \%$ and $65 \%$ of maximal oxygen uptake (12). The exercise intensity, at which 'maximal fat oxidation rate' $\left(\mathrm{Fat}_{\max }\right)$ occurs, has been defined as $\mathrm{Fat}_{\max }$ intensity. Thus, the $\mathrm{Fat}_{\max }$ intensity would maximize the beneficial effects of exercise. According to the published data, muscle fatty acid oxidative capacity is a determinant of whole body fat oxidation during physical activities (13). However, the implied relationship between FORs and exercise intensity during ageing has still not been clearly investigated.

Therefore, we chose healthy men of various ages who did not suffer from metabolism disorders to assess fat oxidation, as determined using a cycle-ergometer during graded incremental exercise, and muscle mass tested by DXA. The purpose of this study was to identify the association between FORs and exercise intensity in the context of age-related muscle loss. The results of this study will contribute to the development of an effective exercise regimen for elderly men to achieve Fat $_{\max }$. We present the following article in accordance with the STROBE reporting checklist (available at http://dx.doi.org/10.21037/apm-20-611).

\section{Methods}

\section{Subjects}

The clinical study was approved by the Ethics Committee of the First Affiliated Hospital of Nanjing Medical University, Jiangsu, China (No.2019-SR-481), in accordance with the Declaration of Helsinki (as revised in 2013). Written informed consents were obtained from all participants. Subjects were excluded if they were weight unstable (gained $>2.0 \mathrm{~kg}$ in the previous 6 months), or had chronic disease (e.g., renal, hepatic, pulmonic, cardiovascular disease or cancer). Ultimately, 224 healthy Chinese men, aged 23-92 years, were recruited for this study. 121 were given a graded incremental exercise test. The remaining were either lost on follow-up, did not provide consent or were unable to tolerate the exercise intensity experiment. Fasting levels of glycated hemoglobin, cholesterol, triglycerides, low-density lipoprotein, and high-density lipoprotein were obtained.

\section{Biochemical analysis}

After overnight fasting, blood samples of participants were obtained and centrifuged at $4.0^{\circ} \mathrm{C}$ for $10 \mathrm{~min}$ at $1,000 \mathrm{rpm}$ and subsequently analyzed. Plasma glucose was determined using the YSI 2300 STAT Plus glucose oxidase assay (Yellow Springs Instruments, Yellow Springs, OH, USA). Serum insulin was measured using a radioimmunoassay (EMD Millipore, Billerica, MA, USA). Serum triglycerides (TG) and cholesterol were analyzed using enzymatic methods with an automated platform (Roche Modular Diagnostics, Indianapolis, IN, USA). Serum triiodothyronine (FT3), thyroxine (FT4), and thyroid stimulating hormone (TSH) levels were measured using the Abbott AxSYM Immunoassay system (Abbott Laboratories, Abbott Park, IL, USA) with intra- and inter-assay coefficients of variation of $<10 \%$ for all measurements.

\section{Measurement of body composition}

Total and regional (trunk, android, gynoid, limbs) fat and lean tissue masses were measured using a Hologic Discovery A (Hologic Inc., Bedford, MA, USA) and analyzed by Encore Software 11. Trunk fat and lean masses were designated from the pelvis cut (lower boundary) to the neck cut (upper boundary). Appendicular skeletal muscle mass (ASM) was calculated as the sum of the muscle masses of the arms and legs.

\section{Measuring rest energy expenditure (REE) by indirect calorimetry (IC)}

The participants visited the metabolic laboratory between 07:00 am and 11:00 am after a $12 \mathrm{~h}$ fast and abstaining from any form of physical activity (other than walking) prior to completing a ventilated, open-circuit IC measurement (Quark PFT Ergo, COSMED SRL, Rome, Italy). The principle of IC is derived from the fact that the human body burns available sources of fuel using oxygen while producing $\mathrm{CO}_{2}$. Only stable measurements for at least 20 minutes were considered acceptable. Oxygen consumption and $\mathrm{CO}_{2}$ production were measured and the respiratory quotient (RQ) 
and REE calculated using the VCO2-based Weir equation $\left(\mathrm{REE}=5.534 \times \mathrm{V}_{\left.\mathrm{CO}_{2} \times 1440\right)}\right.$ (14). To ensure quality control between participants and technicians, gas analyzers were calibrated to $<0.1 \%$ standard gas and flow rate and volume were calibrated to $\leq 3 \%$ error.

\section{Graded exercise protocol and energy expenditure}

The experimental trial was performed in the morning after a minimum 10-h overnight fast. All subjects were asked to refrain from exercise, alcohol, smoking, and caffeine for the $24 \mathrm{~h}$ period preceding the test. After a 15 -min seated resting period, the subjects were seated on the cycle-ergometer and given a graded incremental test. After a standardized 5.0-min warmup at a 0.0 -Watts $(\mathrm{W})$, the intensity was increased by $10 \mathrm{~W}(10 \mathrm{~W}-20 \mathrm{~W}-30 \mathrm{~W}-40 \mathrm{~W}-50 \mathrm{~W}-\ldots)$ every $5 \mathrm{~min}$ until the exercise heart rates of the subjects achieved $70 \%$ of the age-predicted maximum (220-age), which was defined as hard-intensity exercise. The real-time energy consumption, respective contribution (\%) of fat to total energy expenditure were determined using indirect calorimetry (Quark PFT Ergo, COSMED SRL, Rome, Italy). Fat $_{\max }$ intensity is the exercise intensity that causes the highest rate of fat oxidation, which is usually considered to occur at $45-60 \%$ of peak maximal oxygen uptake, defined as moderate intensity with $50-69 \%$ of maximal heart rate; therefore, after incremental exercise, $\mathrm{Fat}_{\max }$ can be determined using the real-time fat oxidation reporting system of indirect calorimetry.

\section{Statistical analysis}

Descriptive data for the subject characteristics were presented as mean \pm standard deviation (SD). The association between fat oxidation rates, $\mathrm{Fat}_{\max }$ and age, appendicular skeletal muscle mass/Weight (ASM/Wt), total fat mass were determined using Pearson correlation analysis. Multiple linear regression models were further applied for fat oxidation rates analyses, using Age, Exercise intensity and ASM/Wt data. All statistical analyses were performed using IBM SPSS Statistics for Windows (Version 20, IBM Corp, Armonk, NY, USA) and $\mathrm{P}<0.05$ was considered statistically significant.

\section{Results}

\section{Skeletal muscle mass decreased during ageing}

A total of 224 healthy Chinese men were included in the analysis, with data obtained from the baseline visit of a clinical trial. Skeletal muscle is a metabolically active tissue that is critical to maintaining whole-body homeostasis. Recent study reported that ASM/Wt was related with insulin resistance (15), and ASM/Wt was the more appropriate index for sarcopenia (16). Pearson correlation analysis showed that $\mathrm{ASM} / \mathrm{Wt}$ was negatively correlated with the age $(\mathrm{r}=-0.260$, $\mathrm{P}<0.0001$ ) (Table 1, Figure S1). In addition, as shown in Table 1 , ASM/Wt was negatively associated with WHR $(\mathrm{r}=-0.206$, $\mathrm{P}=0.004)$ and total fat mass $(\mathrm{r}=-0.409, \mathrm{P}<0.0001)$. ASM/Wt showed a positive relationship with TG $(\mathrm{r}=0.137, \mathrm{P}=0.04)$ and negative relationship with fasting insulin $(\mathrm{r}=-0.228, \mathrm{P}=0.018)$. However, there was no statistical association between ASM/ Wt and fasting glucose, HDL-C, HAc1\%, 25(OH)VD and thyroid function including FT3, FT4 and TSH.

\section{Increased ASM/Wt improves fat oxidation rate during low-intensity exercise}

Age-related muscle loss is associated with metabolic syndrome and muscle is the major site for fat utilization. Thus, we performed correlation analysis between ASM/ $\mathrm{Wt}$ and fat oxidation rate during an exercise intensity of 10-50 W. The results showed a significantly positive correlation between ASM/Wt and fat oxidation rates during low-intensity exercise $(10 \mathrm{~W}: \mathrm{r}=0.252, \mathrm{P}=0.008$ and $20 \mathrm{~W}$ : $\mathrm{r}=0.199, \mathrm{P}=0.039$; Figure $1 A, B)$, while there was no correlation during moderate exercise $(30-50 \mathrm{~W}: \mathrm{P}>0.05$, Figure 1C,D,E).

\section{No relation between ASM/Wt and fat oxidation rate in the resting state}

Resting energy expenditure (REE) is defined as the amount of calories required by the body at rest during a $24 \mathrm{~h}$ period and represents $70 \%$ to $80 \%$ of the calories used by the body. Pearson correlation analysis revealed no correlation between ASM/Wt and REE (Figure S2A, Supporting information). Moreover, there was no significant association between ASM/Wt and fat oxidation rate (Figure S2B).

\section{Analyses of Fat oxidation rate on Age, ASM/Wt and exercise intensity}

Considering that ageing is associated with impaired muscle mass and quality, we further performed correlation analysis between age and fat oxidation rate during an exercise intensity of 10-50 W. The results showed a significantly 
Table 1 Pearson's correlation between ASM/Wt and clinical parameters

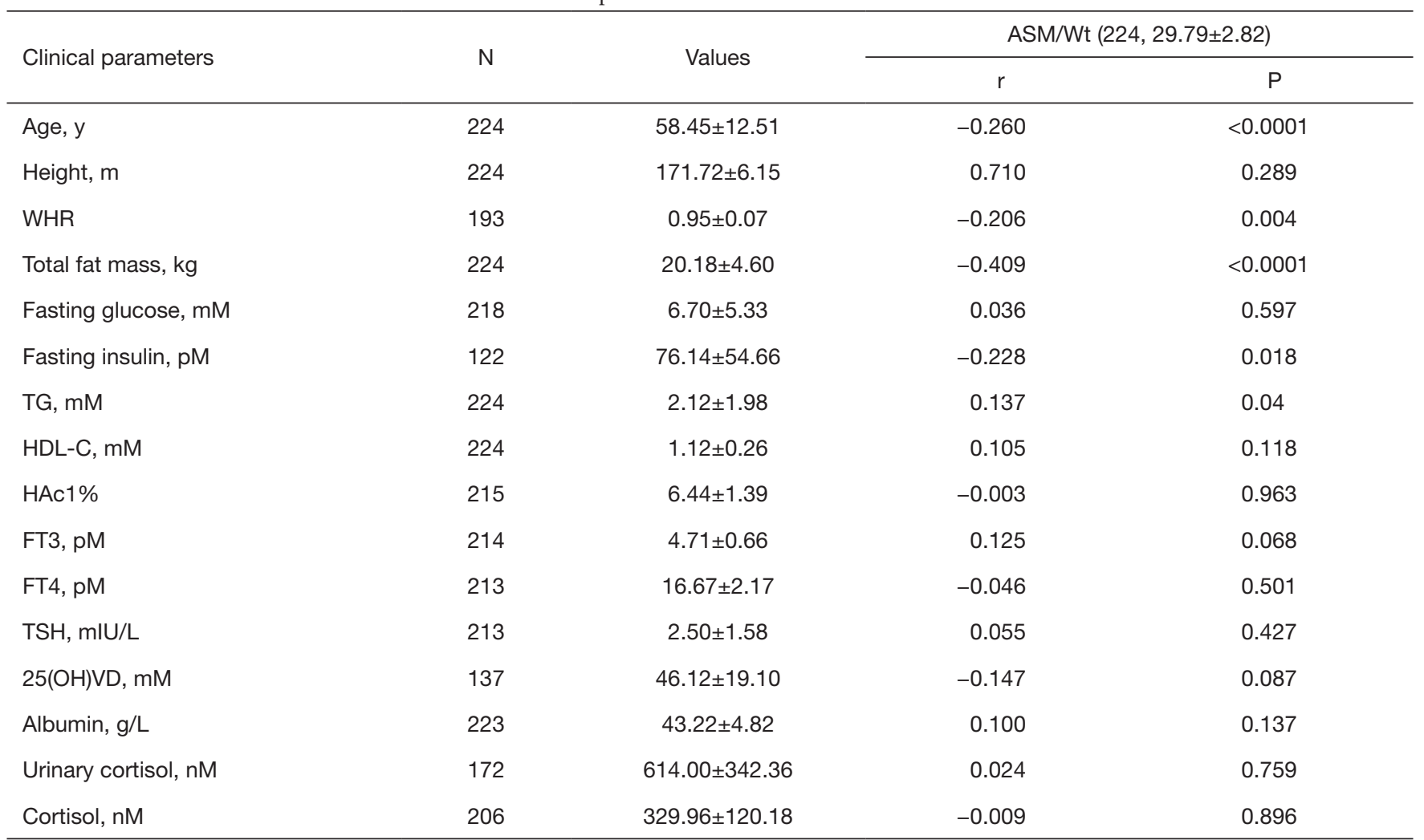

ASM/Wt, appendicular skeletal muscle mass/weight; WHR, waist to hip ratio; TG, triglyceride; HDL-C, high-density lipoprotein cholesterol; HAc1\%, glycated hemoglobin; FT3, triiodothyronine; FT4, thyroxine; TSH, thyroid stimulating hormone; 25(OH)VD, 25 hydroxyvitamin D. Variables are expressed as mean $\pm \mathrm{SD}$. $r$ correlation coefficient. $\mathrm{P}<0.05$ indicates statistically significant difference.

negative correlation between fat oxidation rate and age during low-intensity exercise $(10 \mathrm{~W}: \mathrm{r}=-0.178, \mathrm{P}=0.077$ Figure $2 A ; 20 \mathrm{~W}: \mathrm{r}=-0.202, \mathrm{P}=0.045$ Figure $2 B)$, but not during moderate exercise (30-50 W Figure $2 C, D, E)$.

Given the significant relationship between age, ASM/ $\mathrm{Wt}$, exercise intensity and fat oxidation rate, we further performed multiple linear regression analysis (Table 2). Fat oxidation rate was positively correlated with $\mathrm{ASM} / \mathrm{Wt}$ ( $\beta=0.446, \mathrm{P}=0.0091$ ) but inversely associated with Exercise intensity $(\beta=0.573, \mathrm{P}<0.0001)$, while fat oxidation rate did not show any association with age. Taken together, these results highlight the significance of muscle mass of Chinese men in fat utilization during light-intensity exercise.

\section{The Chinese men with decreased muscle mass need a higher exercise intensity for Fat $_{\text {max }}$}

The subjects were tested using a graded exercise protocol with the intensity being steadily ramped from no load
$(0.0 \mathrm{~W})$ to an increase of $10 \mathrm{~W}$ every 5 min until reaching $\mathrm{Fat}_{\text {max }}$ and the correlation between Fat $\mathrm{max}_{\text {max }}$ and body parameters was determined. The results showed significantly positive correlations of $\mathrm{Fat}_{\max }$ with BMI and total fat $(\mathrm{r}=0.459$, $\mathrm{P}<0.0001$ and $\mathrm{r}=0.348, \mathrm{P}=0.0002$, respectively; Figure $3 A, B$ ), but a negative correlation between $\mathrm{Fat}_{\max }$ and $\mathrm{ASM} / \mathrm{Wt}$ $(\mathrm{r}=-0.244, \mathrm{P}=0.011$; Figure 3C).

\section{Discussion}

Lean muscle mass accounts for about $50 \%$ of total body weight in young adults, but decreases to only $25 \%$ of total body weight in old people aged 75-80 years (17). Evidence from our data also indicated that ASM/Wt was negatively correlated with the age. Moreover, we found that legs lean masses decreased much more than arms lean masses during ageing, consistent with previous observations (18).

Skeletal muscle is a metabolically active tissue that is critical to maintaining whole-body homeostasis. It has 

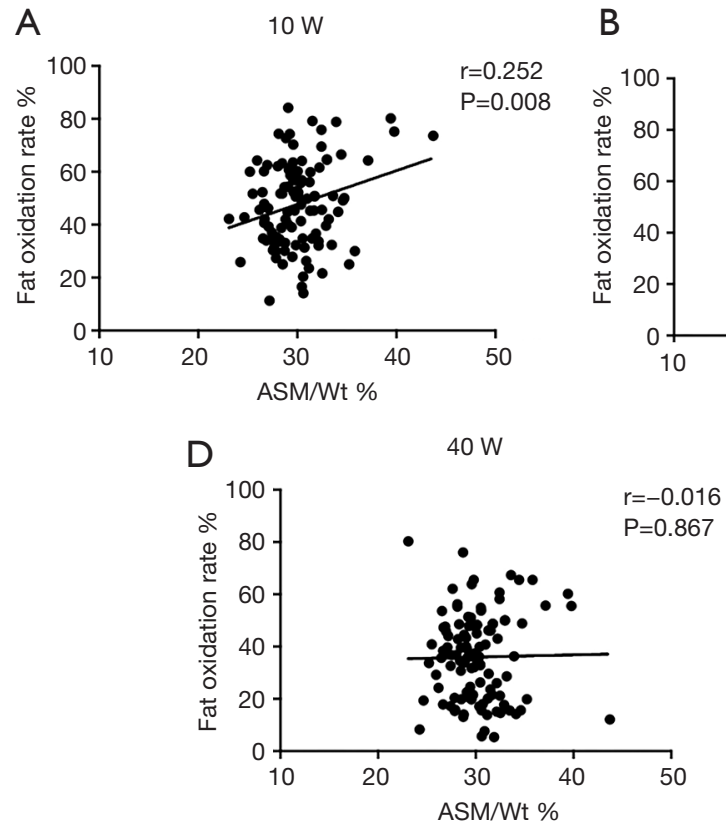

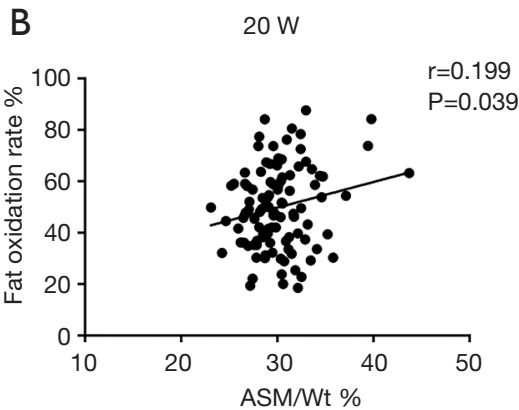

C $\quad 30 \mathrm{~W}$

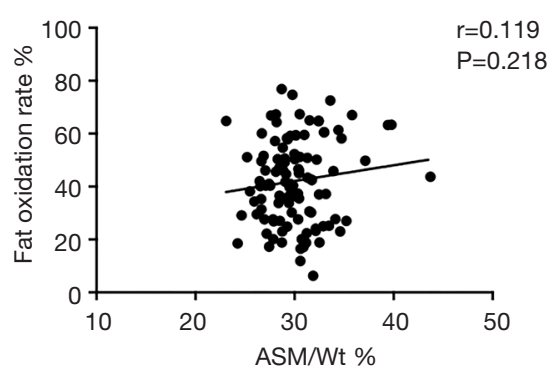

$50 \mathrm{~W}$

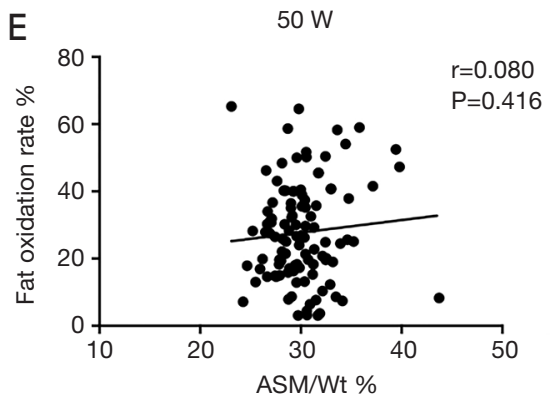

Figure 1 Correlation between appendicular skeletal muscle/Weight (ASM/Wt) mass and fat oxidation rate during exercise. Regression line for ASM/Wt and fat oxidation rate during graded incremental exercise intensity from 10 to $50 \mathrm{~W}$. Pearson $\mathrm{r}$ and $\mathrm{P}$ values are labeled in the top right corner of each graph.
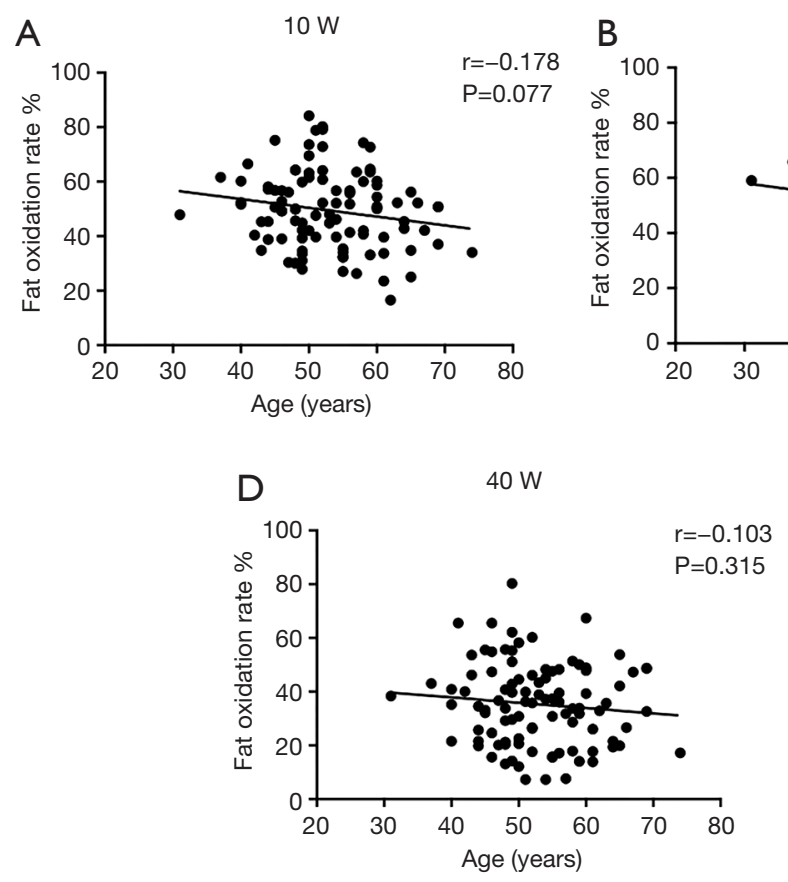
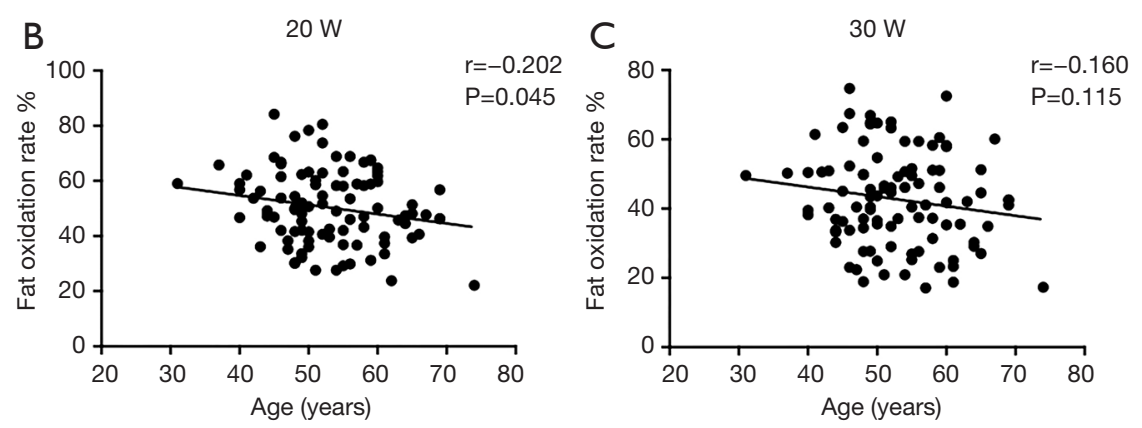

E

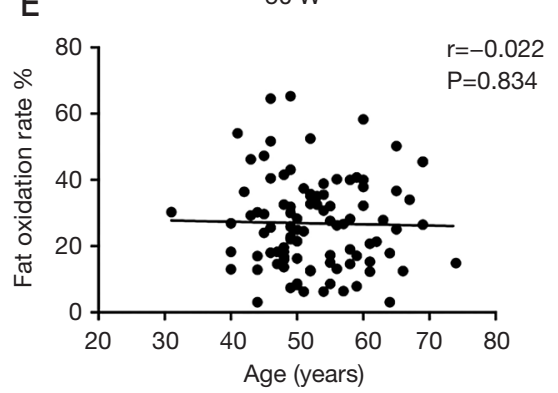

Figure 2 Correlation between age and fat oxidation rate during exercise. Regression line for age and fat oxidation rate during graded incremental exercise intensity from 10 to $50 \mathrm{~W}$. Pearson $\mathrm{r}$ and $\mathrm{P}$ values are labeled in the top right corner of each graph. 
Table 2 Multiple linear regression analyses of fat oxidation rates on age, exercise intensity and appendicular lean skeletal/weight (ASM/Wt)

\begin{tabular}{|c|c|c|c|c|}
\hline Variables & Standardized $\beta$ & Stand error & $t$ & $P$ \\
\hline Age (year) & -0.123 & 0.081 & -1.52 & 0.1301 \\
\hline ASM/Wt (\%) & 0.446 & 0.170 & 2.62 & 0.0091 \\
\hline Exercise intensity (Watts) & -0.573 & 0.046 & -12.52 & $<0.0001$ \\
\hline
\end{tabular}

Adjustment for Age, Exercise intensity and ASM/Wt. $\beta$ standardized coefficient. $\mathrm{P}<0.05$ indicates statistically significant difference.
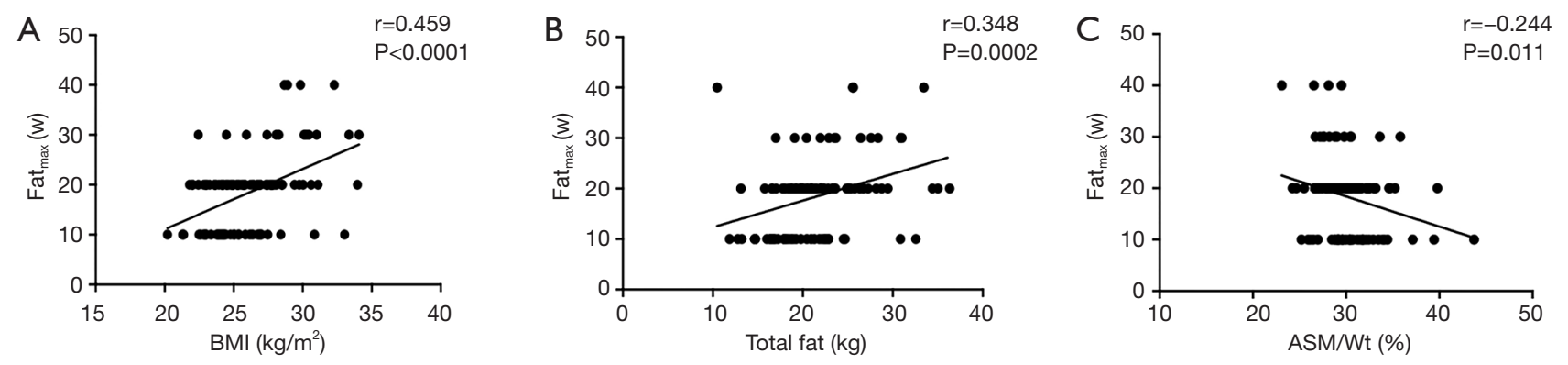

Figure 3 Correlation between body components and maximal fat oxidation rate $\left(\mathrm{Fat}_{\max }\right)$. (A,B,C) Regression line for body mass index (BMI), total fat mass, appendicular lean skeletal/weight (ASM/Wt) and age and Fat ${ }_{\max }$.

been well established that older people have a lower rest energy expenditure (REE) which is decreased by about $30 \%$ between the ages of 20 and 70 years $(19,20)$. Some studies have shown that more muscle mass increases REE (21), but other studies have opposite finding, for example, a study did not demonstrate a low REE in Duchenne Muscular Dystrophy (DMD) males (22). Our present study showed that REE decreased during ageing, but we observed no correlation between ASM/Wt and REE. This might be due to other determinants of REE, such as fat mass, food intake and physical activity habit, which are not considered in this study. It is also possible that skeletal muscle mass plays more important role in the energy expenditure during exercise training than in the resting state.

Moreover, skeletal muscle is the major site for substrate utilization especially during exercise. Carbohydrate $(\mathrm{CHO})$ and fat provide around $90 \%$ of the fuel for skeletal muscle during contraction activity (23). Several studies have shown that the oxidation of lipid contributes significantly to overall energy needs with most of the energy requirements of muscle being obtained via fatty acid oxidative (FAO) $(24,25)$. Fat deposit of muscle has been thought as a strong risk factor of heart disease, type 2 diabetes, and the metabolic syndrome. Furthermore, regular physical activity prevents both the age-associated loss of muscle strength and increase in muscle fat infiltration in older adults (26). Although the mechanisms of muscle loss induced insulin resistance are complex, an inability to oxidize lipids appears to be an important factor (27). An increased capacity to oxidize fat during exercise in patients with T2DM can reduce their dependence on glucose as a source of energy. In addition, increased fat oxidation through physical activity improves insulin sensitivity (28). In our study, we found no statistical association between ASM/Wt and fat oxidation rate and $\mathrm{CHO}$ rate when people are in a state of rest. Nevertheless, we found a significantly positive correlation between ASM/ $\mathrm{Wt}$ and fat oxidation rates during low-intensity exercise $(10 \mathrm{~W}$ and $20 \mathrm{~W})$, indicating that individuals with more muscle mass have an increased capacity to oxidize fat during low-intensity exercise.

In our study, we found that fat oxidation rates do not vary with age. However, we found a significant correlation of fat oxidation rate with muscle mass. This indicates that ageing itself does not affect fat oxidation, but loss of muscle mass during the ageing process does. It is thus important that elderly individuals maintain or increase muscle mass through lifestyle changes such as increased physical activity or better nutrition. Larger muscle mass will lead to higher fat oxidation rates, which will reduce comorbidities and improve quality of life. 
Fat $_{\max }$ intensity is the exercise intensity that causes the highest rate of fat oxidation. Therefore, after incremental exercise, $\mathrm{Fat}_{\max }$ can be determined using the real-time fat oxidation reporting system of indirect calorimetry. Previous studies have demonstrated that $\mathrm{Fat}_{\text {max }}$ intensity is variable among individuals $(29,30)$. For example, A research reported that $\mathrm{Fat}_{\max }$ intensity was higher in obese individuals than in lean controls (31). The exercise intensity for $\mathrm{Fat}_{\max }$ was significantly lower in the T2DM group than the control group (32). Another novel finding of this study was that most of the subjects achieved the Fat ${ }_{\text {max }}$ during a workload of 10-20 W and there was a strong positive correlation between body fat mass and $\mathrm{Fat}_{\max }$ intensity in contrast to a significant negative correlation between muscle mass and $\mathrm{Fat}_{\max }$ intensity. These findings suggest that Chinese men with decreased muscle mass might need more intense exercise to achieve $\mathrm{Fat}_{\max }$.

As the muscle is important for blood glucose homeostasis and increase insulin sensitivity, diabetes and hyperlipidemia are more common in older than in younger individuals. Thus, we examined the relationship of ASM/Wt with multiple clinical variables of glucose and lipid metabolism. We demonstrated that fasting insulin decreased with higher ASM/Wt, suggesting insulin resistance in subjects with loss of skeletal muscle mass. Although strong evidence indicates that individuals with muscle loss have increased risk of insulin resistance, the relationship between muscle loss and lipid metabolism requires further elucidation. For example, several studies indicated a negative association between muscle loss and lipid metabolism biochemical markers $(33,34)$. However, our data showed that the triglycerides level was significantly lower in people with muscle loss. One possible explanation is that not all adipose tissues are metabolically equivalent (35), thus the older people with lighter body weight and without abdominal obesity may contribute to the decline of triglycerides. Meanwhile, ASM/Wt was found to be positively related with FT3. There is increasing evidence that thyroid hormones play a role in muscle loss process (36), for example, in one study hyperthyroid mice exhibited higher muscle fatigue (37).

The large age range required for this study holds specific limitations. For example, there was large variability in parameters such as fasting insulin and cortisol levels between participants. Furthermore, older individuals have different activity levels and dietary habits to their younger counterparts. The study could have been improved if we had acquired data on activity levels and dietary habits, and standardized accordingly. Finally, our study population is small. With a larger population, direct comparisons within age brackets can be made, increasing the applicability of our findings.

In conclusion, our findings highlight the importance of muscle mass in fat utilization during low-intensity exercise. A higher exercise intensity indicated by $\mathrm{Fat}_{\max }$ is recommended for improving fat oxidation in Chinese men with decreased muscle mass. These results could be useful when considering an exercise regimen to improve health in elderly men.

\section{Acknowledgments}

Funding: This study was funded by a grant from the National Natural Science Foundation of China (81871096) to Shan Lv.

\section{Footnote}

Reporting Checklist: The authors have completed the STROBE reporting checklist. Available at http://dx.doi. org/10.21037/apm-20-611

Data Sharing Statement: Available at http://dx.doi. org/10.21037/apm-20-611

Peer Review File: Available at http://dx.doi.org/10.21037/ apm-20-611

Conflicts of Interest: All authors have completed the ICMJE uniform disclosure form (available at http://dx.doi. org/10.21037/apm-20-611). The authors have no conflicts of interest to declare.

Ethical Statement: The authors are accountable for all aspects of the work in ensuring that questions related to the accuracy or integrity of any part of the work are appropriately investigated and resolved. The clinical study was approved by the Ethics Committee of the First Affiliated Hospital of Nanjing Medical University, Jiangsu, China (No.2019-SR-481), in accordance with the Declaration of Helsinki (as revised in 2013). Written informed consents were obtained from all participants.

Open Access Statement: This is an Open Access article distributed in accordance with the Creative Commons Attribution-NonCommercial-NoDerivs 4.0 International License (CC BY-NC-ND 4.0), which permits the non- 
commercial replication and distribution of the article with the strict proviso that no changes or edits are made and the original work is properly cited (including links to both the formal publication through the relevant DOI and the license). See: https://creativecommons.org/licenses/by-nc-nd/4.0/.

\section{References}

1. Rolfe DF, Brown GC. Cellular energy utilization and molecular origin of standard metabolic rate in mammals. Physiol Rev 1997;77:731-58.

2. Frontera WR. Physiologic Changes of the Musculoskeletal System with Aging: A Brief Review. Phys Med Rehabil Clin N Am 2017;28:705-11.

3. Kalyani RR, Corriere M, Ferrucci L. Age-related and disease-related muscle loss: the effect of diabetes, obesity, and other diseases. Lancet Diabetes Endocrinol 2014;2:819-29.

4. Gallagher D, Belmonte D, Deurenberg P, et al. Organtissue mass measurement allows modeling of REE and metabolically active tissue mass. Am J Physiol 1998;275:E249-58.

5. Horton ES. Metabolic fuels, utilization, and exercise. Am J Clin Nutr 1989;49:931-2.

6. Scott D, Trbojevic T, Skinner E, et al. Associations of calf inter- and intra-muscular adipose tissue with cardiometabolic health and physical function in community-dwelling older adults. J Musculoskelet Neuronal Interact 2015;15:350-7.

7. Ribeiro MBT, Guzzoni V, Hord JM, et al. Resistance training regulates gene expression of molecules associated with intramyocellular lipids, glucose signaling and fiber size in old rats. Sci Rep 2017;7:8593.

8. Lanzi S, Codecasa F, Cornacchia M, et al. Short-term HIIT and Fat max training increase aerobic and metabolic fitness in men with class II and III obesity. Obesity (Silver Spring) 2015;23:1987-94.

9. Spriet LL. Regulation of skeletal muscle fat oxidation during exercise in humans. Med Sci Sports Exerc 2002;34:1477-84.

10. Jeukendrup AE, Saris WH, Wagenmakers AJ. Fat metabolism during exercise: a review. Part I: fatty acid mobilization and muscle metabolism. Int J Sports Med 1998;19:231-44.

11. van Loon LJ, Greenhaff PL, Constantin-Teodosiu D, et al. The effects of increasing exercise intensity on muscle fuel utilisation in humans. J Physiol 2001;536:295-304.

12. Romijn JA, Coyle EF, Sidossis LS, et al. Regulation of endogenous fat and carbohydrate metabolism in relation to exercise intensity and duration. Am J Physiol 1993;265:E380-91.

13. Morio B, Hocquette JF, Montaurier C, et al. Muscle fatty acid oxidative capacity is a determinant of whole body fat oxidation in elderly people. Am J Physiol Endocrinol Metab 2001;280:E143-9.

14. Mehta NM, Smallwood CD, Joosten KF, et al. Accuracy of a simplified equation for energy expenditure based on bedside volumetric carbon dioxide elimination measurement--a two-center study. Clin Nutr 2015;34:151-5.

15. Moon SS. Low skeletal muscle mass is associated with insulin resistance, diabetes, and metabolic syndrome in the Korean population: the Korea National Health and Nutrition Examination Survey (KNHANES) 2009-2010. Endocr J 2014;61:61-70.

16. Kim KM, Jang HC, Lim S. Differences among skeletal muscle mass indices derived from height-, weight-, and body mass index-adjusted models in assessing sarcopenia. Korean J Intern Med 2016;31:643-50.

17. Beaudart C, Rizzoli R, Bruyere O, et al. Sarcopenia: burden and challenges for public health. Arch Public Health 2014;72:45.

18. Janssen I, Heymsfield SB, Wang ZM, et al. Skeletal muscle mass and distribution in 468 men and women aged 18-88 yr. J Appl Physiol (1985) 2000;89:81-8.

19. Bosy-Westphal A, Eichhorn C, Kutzner D, et al. The agerelated decline in resting energy expenditure in humans is due to the loss of fat-free mass and to alterations in its metabolically active components. J Nutr 2003;133:2356-62.

20. Bosy-Westphal A, Kossel E, Goele K, et al. Contribution of individual organ mass loss to weight loss-associated decline in resting energy expenditure. Am J Clin Nutr 2009;90:993-1001.

21. Zurlo F, Larson K, Bogardus C, et al. Skeletal muscle metabolism is a major determinant of resting energy expenditure. J Clin Invest 1990;86:1423-7.

22. Zanardi MC, Tagliabue A, Orcesi S, et al. Body composition and energy expenditure in Duchenne muscular dystrophy. Eur J Clin Nutr 2003;57:273-8.

23. Jeukendrup AE. Modulation of carbohydrate and fat utilization by diet, exercise and environment. Biochem Soc Trans 2003;31:1270-3.

24. Deriaz O, Dumont M, Bergeron N, et al. Skeletal muscle low attenuation area and maximal fat oxidation rate during submaximal exercise in male obese individuals. Int J Obes Relat Metab Disord 2001;25:1579-84. 
25. Gomes MJ, Martinez PF, Pagan LU, et al. Skeletal muscle aging: influence of oxidative stress and physical exercise. Oncotarget 2017;8:20428-40.

26. Goodpaster BH, Chomentowski P, Ward BK, et al. Effects of physical activity on strength and skeletal muscle fat infiltration in older adults: a randomized controlled trial. J Appl Physiol (1985) 2008;105:1498-503.

27. Chabi B, Ljubicic V, Menzies KJ, et al. Mitochondrial function and apoptotic susceptibility in aging skeletal muscle. Aging Cell 2008;7:2-12.

28. Goodpaster BH, Katsiaras A, Kelley DE. Enhanced fat oxidation through physical activity is associated with improvements in insulin sensitivity in obesity. Diabetes 2003;52:2191-7.

29. Brun JF, Malatesta D, Sartorio A. Maximal lipid oxidation during exercise: a target for individualizing endurance training in obesity and diabetes? J Endocrinol Invest 2012;35:686-91.

30. Achten J, Jeukendrup AE. Maximal fat oxidation during exercise in trained men. Int J Sports Med 2003;24:603-8.

31. Jabbour G, O'Loughlin J, Sabiston C, et al. Increased lipid oxidation during exercise in obese pubertal girls: a QUALITY study. Obesity (Silver Spring) 2014;22:E85-90.
32. Suk MH, Moon YJ, Park SW, et al. Maximal Fat Oxidation Rate during Exercise in Korean Women with Type 2 Diabetes Mellitus. Diabetes Metab J 2015;39:328-34.

33. O'Brien LC, Chen Q, Savas J, et al. Skeletal muscle mitochondrial mass is linked to lipid and metabolic profile in individuals with spinal cord injury. Eur J Appl Physiol 2017;117:2137-47.

34. Perna S, Guido D, Grassi M, et al. Association between muscle mass and adipo-metabolic profile: a crosssectional study in older subjects. Clin Interv Aging 2015;10:499-504.

35. Kang SM, Yoon JW, Ahn HY, et al. Android fat depot is more closely associated with metabolic syndrome than abdominal visceral fat in elderly people. PLoS One 2011;6:e27694.

36. Bloise FF, Oliveira TS, Cordeiro A, et al. Thyroid Hormones Play Role in Sarcopenia and Myopathies. Front Physiol 2018;9:560.

37. Elnakish MT, Schultz EJ, Gearinger RL, et al. Differential involvement of various sources of reactive oxygen species in thyroxin-induced hemodynamic changes and contractile dysfunction of the heart and diaphragm muscles. Free Radic Biol Med 2015;83:252-61.
Cite this article as: Chen L, Tang T, Liu J, Yu J, Di W, Li Y, Lv S. Low muscle mass impairs fat utilization during light-intensity exercise in Chinese men. Ann Palliat Med 2020;9(4):1453-1461. doi: 10.21037/apm-20-611 


\section{Supplementary}

A

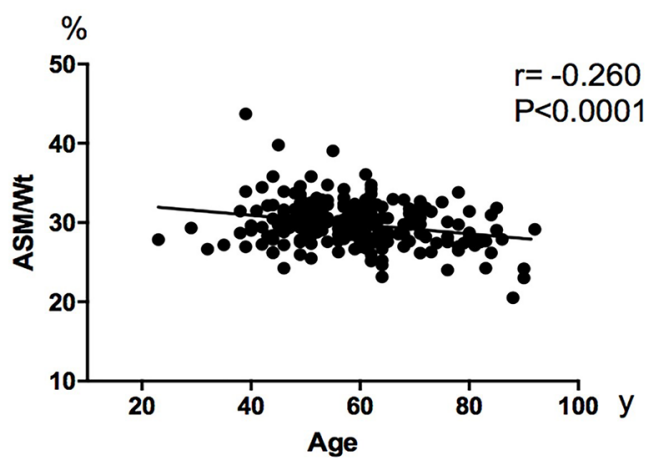

B $\mathrm{kg}$

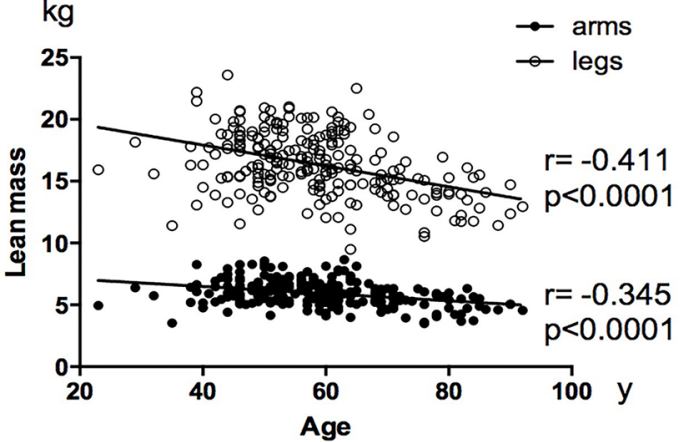

Figure S1 Pearson's correlation between age and appendicular skeletal muscle mass/weight (ASW/Wt) and lean mass. (A) age correction with ASM/Wt. (B) age correction with Lean mass (arms and legs).
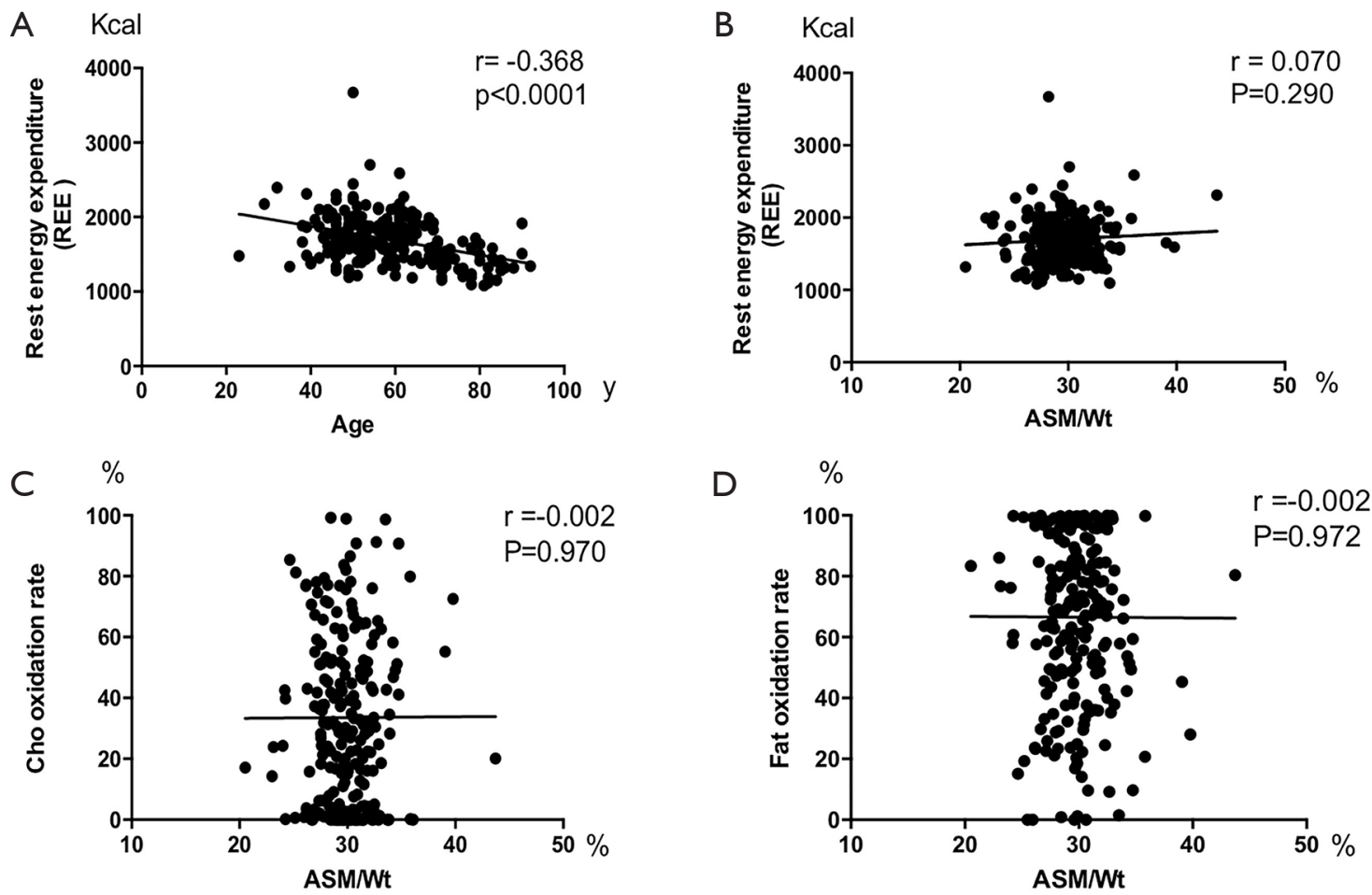

Figure S2 Pearson's correlation between rest energy expenditure (REE) and age and relation with appendicular skeletal muscle mass/weight (ASW/Wt) during a rest state. (A) age correlation with REE. (B,C,D) ASW/Wt correction with REE, cho oxidation rate and fat oxidation rate 